\title{
Convergence of external Crohn's disease risk factors on intestinal bacteria
}

\author{
Alexander Oberc ${ }^{1,2}$ and Brian K. Coombes , $^{1,23 *}$ \\ ${ }^{1}$ Department of Biochemistry and Biomedical Sciences, McMaster University, Hamilton, ON, Canada, ${ }^{2}$ Michael G. DeGroote \\ Institute for Infectious Disease Research, Hamilton, ON, Canada, ${ }^{3}$ Farncombe Family Digestive Health Research Institute, \\ Hamilton, ON, Canada
}

Crohn's disease (CD) is an immune-mediated intestinal illness that significantly compromises health in many developed countries. Although definitive causes remain elusive, the required contribution of microbes in the progression of disease has become an accepted concept. Known CD risk factors, such as antibiotic use and acute infectious gastroenteritis, may impact the gut. This concept is now being explored with a view toward understanding the beneficial and unfavorable microbes that may be altered in numbers during such external insults. A comprehensive understanding of the microbial component to CD could be useful clinically as future therapies may focus on preventing

OPEN ACCESS

Edited by:

Amélia M. Sarmento,

Universidade Fernando Pessoa,

Portugal

Reviewed by:

Emma Slack,

ETH Zürich, Switzerland

Pam Kozlowski,

Louisiana State University Health

Sciences Center, USA

*Correspondence:

Brian K. Coombes

coombes@mcmaster.ca

Specialty section:

This article was submitted to Mucosal

Immunity, a section of the

journal Frontiers in Immunology

Received: 14 August 2015

Accepted: 19 October 2015

Published: 03 November 2015

Citation:

Oberc A and Coombes BK (2015)

Convergence of external Crohn's

disease risk factors on

intestinal bacteria.

Front. Immunol. 6:558.

doi: 10.3389/fimmu.2015.00558 risk exposures on susceptible individuals, eliminating harmful microbes, or restoring a protective gut microbiome. Here, we examine how acute infectious gastroenteritis and antibiotic exposure may impact the gut microbiota in the context of inflammation in CD.

Keywords: Crohn's disease, adherent-invasive E. coli, gastroenteritis, antibiotics, microbiota

\section{INTRODUCTION}

Crohn's disease (CD) is part of the inflammatory bowel disease spectrum that can affect any portion of the gastrointestinal tract. CD is becoming increasingly common in many high-income nations, particularly among the pediatric population (1). Due to its high prevalence and chronic disease course, $\mathrm{CD}$ imposes significant directs costs on the health care system, along with a high attendant societal cost (2). Since the classification of CD in the 1950s, significant investments have been made in understanding the root causes of the disease, as well as in new treatments to induce remission that target mainly the aberrant inflammatory response underlying the disease course. Although genetics are known to play an important role in $\mathrm{CD}(3,4)$, penetrance is low for any one genetic risk allele. Furthermore, the sharp rise in $\mathrm{CD}$ in many countries over the past 40 years indicates that genetics alone does not fully explain the etiology of disease, but rather that environmental exposures may be antecedent to disease initiation and progression in a genetically susceptible population (5). Understanding the nature and origins of such environmental risk factors is of paramount importance in curbing the rise of $\mathrm{CD}$ worldwide.

Clinical and animal studies have provided robust data indicating that intestinal microorganisms, both luminal and tissue-associated, play an important role in CD pathogenesis (5-9). For example, a pathogenic role for luminal bacteria is consistent with the beneficial effects of experimental fecal stream diversion following ileocolonic resection (10) and levels of epithelial-adherent bacteria have been found to correlate clinically with CD severity $(11,12)$. Environmental exposures, such as infectious agents or use of certain xenobiotics, can create intestinal vulnerabilities, rendering one more 
susceptible to the functional and microbial changes associated with $\mathrm{CD}$. In this review, we briefly summarize the microbial composition changes that have been described in CD patients, and discuss two CD risk factors - acute infectious gastroenteritis and exposure to antimicrobial agents - that are known to induce pervasive changes to the microbial composition in the gut. The potential for dysbiosis-inducing external insults such as these to select for the expansion of pathobiont-like bacteria that affect the inflammatory tone of the gut is discussed.

\section{MICROBIAL DYSBIOSIS IN CROHN'S DISEASE}

The advent of high-throughput sequencing has allowed researchers to investigate the gut microbiome in health and disease states, which was previously difficult to study due to the immense quantity and diversity of species present as well as the difficulty in getting broad representation of the intestinal community by culturing methods (13). Several high-throughput methods have been developed over the past decade that have allowed researchers to identify intestinal bacteria based on genetic signatures. One method involves using fluorescence in situ hybridization (FISH) probes designed to label certain groups of bacteria. These labeled bacteria can then be quantified using flow cytometry (FCM-FISH) (14). This method has the advantage of being relatively simple and inexpensive; however, the FCM-FISH method has relatively poor resolution and can only identify broad changes in bacterial groups. High-throughput sequencing has set a new benchmark, allowing researchers to analyze the population structure of complex microbial communities (15). In this regard, 16s rRNA is a useful genetic marker as it is universally present among all bacteria. It contains both variable regions useful for genomic classification and conserved regions that can be used to design universal primers. The choice of primers is extremely important, as even "universal" primers may poorly amplify some bacterial families. Although sequencing larger segments of the 16s rRNA region provides more resolution, most high-throughput sequencing technologies are limited to sequencing several hundred base pairs. For this reason, most studies analyze only one or several variable regions of the 16s rRNA gene. Nonetheless, even short reads of variable regions around 100 bases are usually sufficient to differentiate many microbes to the genus level, although using different variable regions can lead to different results (16). When comparing the results of 16s rRNA sequencing, it is important to consider differences in primer choice and the length of DNA sequenced from each fragment. Additionally, differences in data clean up as well as data analysis can lead to additional variability. For example, different analysis procedures between groups can result in considerable discrepancies in data generation and interpretation that should be taken into consideration when comparing observations from different publications. Nevertheless, some themes are emerging from the literature, which are discussed below.

The human microbiome is composed primarily of anaerobes within the bacterial phyla Firmicutes and Bacteriodetes. One of the first studies to investigate the $\mathrm{CD}$ microbiome examined fecal bacteria from six healthy individuals and six CD patients using both 16s rRNA sequencing and FCM-FISH (17). All CD patients were in remission, had no exposure to antibiotics for at least 3 months, and had previous ileal involvement in the disease course. In this study, CD patients had much lower microbial diversity than the control group, with 88 ribotypes represented in healthy subjects compared to only 54 in CD patients. This change was largely due to decreased diversity in the Firmicutes phylum, in particular, the Clostridium leptum group. C. leptum are known to produce butyrate, a short chain fatty acid (SCFA) and energy source for the intestinal epithelium. Butyrate has also been shown to down regulate pro-inflammatory mRNA within enterocytes and to acidify the intestinal lumen that is thought to inhibit certain pathogens such as Salmonella and Escherichia coli (18, 19). A similarly designed study using FCM-FISH analyzed fecal samples from $14 \mathrm{CD}$ patients and 13 healthy controls that had not received sulfasalazine, antibiotics, or laxatives in the month prior to analysis (20). Using FISH with group-specific probes the study also found that C. leptum was significantly decreased in the CD group.

A larger study examined intestinal biopsy samples rather than fecal samples, from CD and UC patients and healthy controls $(n=190)$ using 16s rRNA sequencing (21). Bacteroidetes and Lachnospiraceae groups were greatly decreased in these subjects, whereas Actinobacteria and Proteobacteria groups were increased. Another study investigated both fecal and mucosal samples from healthy $(n=27), \mathrm{CD}(n=121)$, and UC patients $(n=75)$ utilizing 16 s rRNA sequencing of V3-V5 regions (22). Roseburia, Phascolarctobacterium, and Ruminococcaceae were all reduced in CD patients. These microbial groups are also important producers of SCFA. In ileal CD patients, Ruminococcaceae and Faecalibacterium groups were particularly reduced while Escherichia/Shigella species were enriched, a finding that has been reproduced in several studies. Interestingly, the use of 5-ASA was strongly correlated with a decrease in Escherichia/Shigella. Various genera within Clostriales order were decreased in patients treated with antibiotics, possibly due to their sensitivity to ciprofloxacin/metronidazole often used in CD treatment. The genes involved in several major metabolic pathways have been well characterized in model bacteria. Since many of the genome of gut microbes have been sequenced, it is possible to predict if a microbe lacks a particular classical pathway based on an absence of conservation to known genes typically involved in that pathway. Overall, microbes from IBD patients had decreased pathways for amino acid synthesis coupled with increased amino acid uptake pathways. There were also increased levels of carbohydrate and lipid uptake and metabolism pathways, particularly in ileal CD. Many of these changes may aid in the metabolism of mucin, which is often overproduced during intestinal inflammation. The reduction in amino acid synthesis resembles phenotypes elicited by murine pathobionts known as segmented filamentous bacteria $(23,24)$. The microbiome showed increased glutathione uptake, which may be important for surviving oxidative stress from inflammation (25). Finally, the ileal CD metagenome was enriched in bacterial secretion systems, in particular, the type II secretion system that is often used in the export of toxins (26).

Another recent study analyzed the treatment-naïve microbiome of pediatric CD patients $(n=447)$ and healthy controls 
( $n=221$ ) using tissue biopsies and feces and sequencing the 16s rRNA V4 region (27). When comparing intestinal biopsy samples from healthy individuals to $C D$ patients, the Bacteroidales and several species within the C. leptum groups had lower abundance in pediatric CD patients in accordance with previous studies. Several bacterial families that were found to be more abundant were Enterobacteriaceae, Pasteurellaceae, Veillonellaceae, Neisseriaceae, and Fusobacteriaceae. The use of antibiotics in patients already diagnosed with CD is controversial $(28,29)$. However, in this study, a subset of CD patients had been treated with antibiotics providing for a separate analysis. When this group was analyzed separately, it was found that antibiotic exposure amplified the microbial dysbiosis associated with CD. Importantly, in new onset patients, the microbial dysbiosis was poorly reflected in the stool compared to tissue biopsy samples, which might suggest that the bacterial community of pathogenic significance in the disease is tissue associated.
Overall, these studies consistently show that CD is associated with decreased abundance and diversity of the Firmicutes phylum with an increased abundance in the Proteobacteria phylum (Figure 1). Tissue samples appear to be more indicative of dysbiosis compared to fecal samples at least in early disease. Although Firmicutes appear decreased in overall abundance in $\mathrm{CD}$, some specific members of the Firmicutes are conversely increased. These studies occasionally differ on the particular groups of bacteria that are elevated or reduced in $\mathrm{CD}$ patients. These differences may be due to differences in sample type (luminal vs. tissue) as well as differences in patient populations (patients with active disease vs. remission, treated patients vs. treatment naïve), or in method of analysis. How these shifts in microbial ecology contribute to the pathophysiology of $\mathrm{CD}$ remains an active area of research. A recent study using a murine model of CD demonstrated that bacteria gut dysbiosis played a causal role in intestinal inflammation (30). Several mechanisms may explain why dysbiosis is associated with CD. It is possible

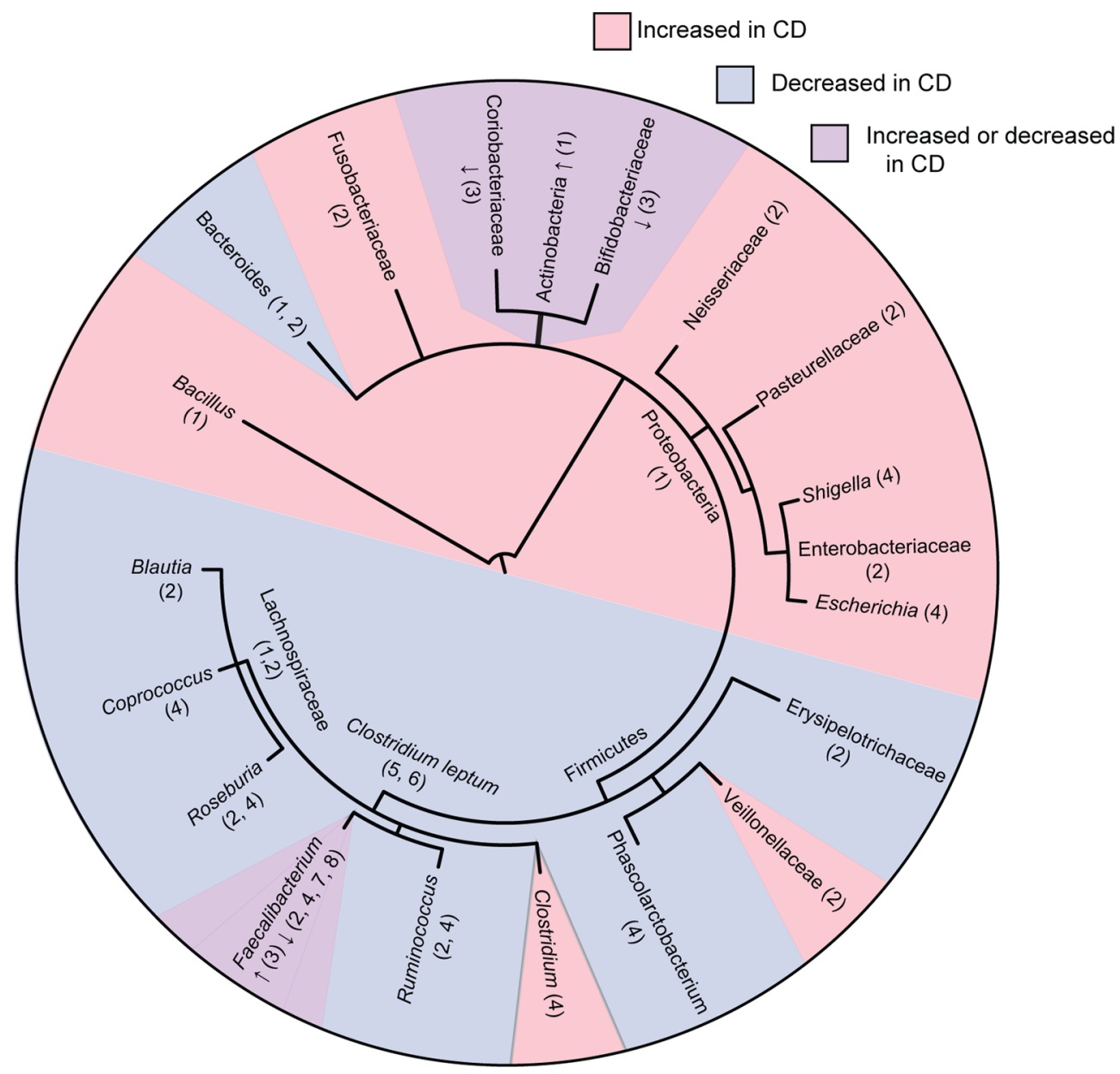

FIGURE 1 | Phylogenetic tree of bacterial groups associated with increased or decreased abundance in the gut microbiome of Crohn's disease patients. In cases were bacterial groups have been associated both increases and decreases, studies showing increased abundance are indicated with " $\uparrow$ " and studies showing decreased abundance are indicated with ". ." Numbers indicate references as follows; 1, Frank et al. (21); 2, Gevers et al. (27); 3, Hansen et al. (31); 4, Morgan et al. (22); 5, Sokol et al. (20); 6, Manichanh et al. (17); 7, Sokol et al. (32); 8, Sokol et al. (33). Tree topology was created using phyloT (http://phylot. biobyte.de/) and the figure was made using ITOL (http://itol.embl.de/). 
that dysbiosis contributes to CD because the loss of SCFA producing bacteria has multiple impacts on the gut environment, including impaired survival of enterocytes, increased production of inflammatory cytokines, and decreased suppression of potentially pathogenic Proteobacteria. It is also possible that dysbiosis is caused by the intestinal inflammation in CD. Bacterial species from Proteobacteria are facultative anaerobes that tend to have a higher resistance to reactive-oxygen species produced during inflammation, possibly giving them a selective advantage over the predominantly obligate anaerobes from Firmicutes and Bacteroidetes. These mechanisms are not mutually exclusive, and it is likely that microbial dysbiosis both contributes to and results from the intestinal inflammation seen in CD.

One member of the Firmicutes that has received much attention in the context of CD is Faecalibacterium prausnitzii, a major member of the C. leptum group of Firmicutes that is decreased in CD patients (32). In particular, a higher abundance of $F$. prausnitzii was associated with a higher rate of remission following resection surgery (33). In vitro analysis showed that this bacterium was capable of inhibiting $\mathrm{NFKB}$ and the secretion of pro-inflammatory cytokines, as well as stimulating the release of anti-inflammatory cytokines, such as IL-10. In vivo studies using dinitrobenzene sulfonic acid (DNBS) (34) and trinitrobenzenesulfonic acid (TNBS) (33)-induced colitis models showed that $F$. prausnitzii and its culture supernatant were capable of attenuating inflammation when introduced both orally and into the peritoneal cavity. An intriguing recent study has identified a protein secreted by $F$. prausnitzii that may explain its anti-inflammatory properties (35). However, the exact role of $F$. prausnitzii in the pathogenesis of CD has yet to be elucidated. Two studies of pediatric patients with newly diagnosed, untreated CD conflictingly showed increased (31) and decreased (27) levels of in F. prausnitzii compared to healthy controls. It is possible that the decreased levels of F. prausnitzii are a characteristic of adult CD but not pediatric CD. It is also possible that F. prausnitzii is lost due to medications used to treat CD such as antibiotics rather than having a direct link to disease expression. Additional research is needed to determine how levels of F. prausnitzii might dictate the fine balance between health and disease in the gut.

\section{INFECTIOUS GASTROENTERITIS AS A RISK FACTOR FOR CD}

Amply powered clinical studies show that acute infectious gastroenteritis caused by enteric pathogens (including Salmonella and pathogenic E. coli) increases the short- and long-term risk of developing CD (36-38) (Table 1). In one study, Garcia-Rodríguez et al. compared a cohort of individuals with acute gastroenteritis $(N=43,013)$ with matched controls without documented gastroenteritis $(N=50,000)$, excluding individuals with previous IBD diagnosis (36). Over a mean follow-up period of 3.5 years, there was an increased risk of developing IBD after a single episode of acute gastroenteritis (HR 2.4 [1.7-3.3]). The association was stronger with CD compared to ulcerative colitis (UC), particularly in the first year after gastroenteritis (HR 6.6 [1.9-22.4]). A second case-control study by Porter et al. compared individuals diagnosed with CD with healthy controls (37). The study examined previous diagnoses of infectious gastroenteritis in both groups. Patients with IBD were more likely to have had a previous diagnosis of infectious gastroenteritis (AOR 1.40 [1.19-1.66]). Once again, the risk was higher for CD (AOR 1.54 [1.17-2.04]) than for UC (AOR 1.36 [1.08-1.72]). Finally, a retrospective study compared cohorts of healthy individuals in Denmark to those diagnosed with acute gastroenteritis caused by either Salmonella or Campylobacter, tracking subsequent IBD diagnoses over the following 15 years (38). Overall, the gastroenteritis group had an increased risk of subsequent IBD diagnosis over the entire 15-year period (HR 2.9 [2.2-3.9]), with the greatest risk occurring in the first year following an episode of acute gastroenteritis.

Due to the observational nature of these studies, it is difficult to determine whether infection predisposes individuals to $\mathrm{CD}$ or if individuals with a susceptibility $C D$ are more susceptible to infection. It is possible that low-grade inflammation in prodromal CD may lower the colonization resistance to enteric pathogens, a phenomenon has been demonstrated in murine models (39). Several studies have shown that infectious gastroenteritis is associated with microbial dysbiosis, albeit transiently $(40,41)$, that might underlie the microbial triggers of chronic disease. Though the mechanisms are currently unknown, the long-term risk of developing CD following acute infectious gastroenteritis might suggest that resident gut microbes could perpetuate inflammatory reactions in the post-infectious period. One central hypothesis is that exposure to infectious pathogens creates an environment favorable to colonization by other proinflammatory bacteria. These may be existing members of the microbiota typically restricted by microbial and host processes, or they may be acquired de novo through vulnerabilities created by the disruption of the resident microbiota during gastroenteritis. For example, human $(42)$ and animal studies $(43,44)$ indicate that inflammation during gastroenteritis selectively disrupts the resident intestinal microbiota in favor of Enterobacteriacea, such as E. coli. Whether such disruptions have long-term consequences on the host in the post-infectious period has not been rigorously studied.

TABLE 1 | Epidemiological studies associating infectious gastroenteritis and Crohn's disease.

\begin{tabular}{|c|c|c|c|c|c|}
\hline Study & Type & Location & Population size & Type of association & Risk [95\% Cl] \\
\hline Garcia Rodriguez et al. (36) & Retrospective cohort & United Kingdom & IGE: 43,013 Cont: 50,000 & Overall & HR $6.6[1.9,22.4]$ \\
\hline Porter et al. (37) & Case-control & United States & CD: 1,037 Cont: 11,646 & Overall & OR $1.54[1.1,2.04]$ \\
\hline Gradel et al. (38) & Case-control & Denmark & IGE: 13,148 Cont: 26,216 & Overall & HR $3.0[1.7,5.3]$ \\
\hline
\end{tabular}

IGE, infectious gastroenteritis; HR, hazard ratio; OR, odds ratio. 
TABLE 2 | Epidemiological studies associating antibiotic treatment with subsequent CD diagnosis.

\begin{tabular}{|c|c|c|c|c|c|}
\hline Study & Type & Location & $\begin{array}{l}\text { Population size } \\
\text { (CD:control or } \\
\text { cohort size) }\end{array}$ & Type of association & Risk [95\% Cl] \\
\hline Card et al. (49) & $\begin{array}{l}\text { Adult } \\
\text { Case-control }\end{array}$ & United Kingdom & $587: 1460$ & $2-5$ years before CD diagnosis & OR $1.32[1.05,1.65]$ \\
\hline Margolis et al. (50) & $\begin{array}{l}\text { Adult } \\
\text { Retrospective cohort }\end{array}$ & United Kingdom & 94,487 & Overall & HR $1.62[1.04,2.53]$ \\
\hline Shaw et al. (48) & $\begin{array}{l}\text { Adult } \\
\text { Case-control }\end{array}$ & Canada & $1025: 22,346$ & $2-5$ years before $C D$ diagnosis & AOR $1.29[1.18,1.40]$ \\
\hline Hildebrand et al. (53) & $\begin{array}{l}\text { Pediatric } \\
\text { Case-control }\end{array}$ & Sweden & 1098:6550 & Pneumonia $<5$ years old & OR $3.56[1.79,7.08]$ \\
\hline Hviid et al. (51) & $\begin{array}{l}\text { Pediatric } \\
\text { Retrospective cohort }\end{array}$ & Denmark & $577: 627$ & Overall & $\operatorname{RR} 3.41[1.45,8.02]$ \\
\hline Virta et al. (52) & $\begin{array}{l}\text { Pediatric } \\
\text { Case-control }\end{array}$ & Finland & 233:2380 & 2 years before CD diagnosis & AOR $1.87[1.37,2.56]$ \\
\hline
\end{tabular}

$O R$, odds ratio; HR, hazard ratio; $A O R$, adjusted odds ratio; $R R$, relative risk.

\section{ANTIBIOTICS AS A RISK FACTOR FOR CD}

Antibiotics are commonly prescribed for a wide range of illness and are occasionally used in the treatment of CD (45). Antibiotics have long been known to have negative impacts on commensal microbes and are known to be an important risk factor for gastrointestinal illnesses, such as Clostridium difficile infection $(46,47)$. Several epidemiological studies have linked exposure to antibiotics with $\mathrm{CD}$, which are summarized in Table 2. A case-control study in Canada compared antibiotic use in individuals with incident IBD diagnosis and non-IBD controls and found that patients diagnosed with IBD were more likely to have been prescribed antibiotics in the preceding 5 years before diagnosis (48). Of interest, there was a dose-dependent relationship between the number of antibiotics dispensed and the risk of subsequent IBD diagnosis. The risk of subsequent IBD diagnosis was highest 2 years after antibiotics and diminished over time. Nearly all antibiotics included in this study, with the exception of clindamycin, were associated with an increased risk of IBD diagnosis. Interestingly, metronidazole had the highest risk (AOR 2.86 [2.24-3.65]) followed by quinolones (AOR 1.45 [1.27-1.64]). Both of these antibiotics are also used as treatments for CD.

A case-control study using data from the UK examined patient medical history at least 5 years prior to diagnosis of CD (49). Again, patients who were later diagnosed with CD were more likely to have taken antibiotics (OR 1.32 [1.05-1.65]). When individual antibiotics were examined, only metronidazole and tinidazole (OR 1.71 [1.05-2.76]) and tetracylines (OR 1.33 [1.01-1.77]) were associated with subsequent CD. This study did not find a dose-dependent relationship between the numbers of antibiotics dispensed and overall CD risk.

A cohort study from the UK examined the association between oral tetracycline for the treatment acne in adults and adolescents and subsequent IBD diagnosis (50). Once again, there was an association between tetracycline use and IBD (HR 1.39 [1.02-1.90]) and a stronger association with CD in particular (HR 1.62 [1.04-2.53]). Furthermore, the risk of CD varied based on the type of antibiotic, with minocycline conferring the lowest risk (HR 1.28 [0.72-2.29]) followed by tetracycline (HR 1.61 [1.00-2.63]) and finally doxycycline (HR 2.25 [1.27-4.00]).

Several studies in pediatric patients have shown similar results. A study in Denmark followed all singleton children born between 1995 and 2003 (51) and tracked antibiotic prescriptions and IBD diagnoses. CD, but not UC, was associated with previous antibiotic prescriptions (RR 3.41 [1.45-8.0]). This relationship was dose dependent, with the highest risk being among children prescribed $\geq 7$ antibiotics (RR 7.32 [2.14-24.9]). Interestingly, the risk of diagnosis was highest within 3 months of antibiotic exposure (RR 4.43 [1.88-10.44]). Penicillin V and extended spectrum penicillins conferred the highest risk (RR 2.92 [1.22-6.97] and RR 3.13 [1.337.40], respectively). Another study from Finland similarly found an association between antibiotic use 2 years preceding CD (AOR 1.87 [1.37-2.56]), but not UC, diagnosis (52). Cephalosporin antibiotics were associated with the highest risk. Finally, a Swedish study which used childhood infections as a proxy for antibiotic treatment found a position association between pneumonia, diagnosed at $<5$ years old, and subsequent CD (OR 3.56 [1.79-7.08]) (53).

These studies have provided evidence for a correlation between antibiotic use and subsequent diagnosis of CD; however, most of the studies do not agree on a specific class of antibiotic that confers the highest risk, which may relate to differences in patient populations or prescribing practices. Due to the observational nature of these studies, it is difficult to show causality between antibiotics and $\mathrm{CD}$ and it remains possible that the phenotype that makes one susceptible to CD may also make one susceptible to infections requiring antibiotics. Conversely, among patients already diagnosed CD, the use of antibiotics is associated with a reduced chance of flaring (54). Relatively short courses of antibiotics are known to lead to dramatic shifts and loss of diversity on the gut microbiome, which can persist for years (55). It is possible that antibiotic exposure may trigger an unfavorable microbial community structure that increases the potential for long-term dysbiosis and risk of CD in susceptible individuals. Microbiome comparison between $\mathrm{CD}$ patients with and without exposure to antibiotics supports this claim, showing that antibiotics magnify the dysbiosis signature associated with CD (27). Nonetheless, 
robust experimental evidence is needed to clarify the impact of antibiotic use on host susceptibility to disease.

\section{TISSUE-ASSOCIATED BACTERIA ARE INCREASED IN CROHN'S DISEASE}

Inflammation in $\mathrm{CD}$ can involve both the small and large bowel and is accompanied by changes in the microbial composition and distribution at these sites $(6,13)$. Clinical observations are consistent in finding increased numbers of bacteria associated with the intestinal epithelium in Crohn's patients $(5,12,22)$. The intestinal mucosa, made up of the epithelium and underlying lamina propria, is a site rich in immune cell populations that protect the integrity of the epithelial surface and direct the innate and adaptive immune responses. Since this site is consistently breached in $\mathrm{CD}$, attention has focused on the relationship between the innate immune system and the microbiota (56), with a particular emphasis on the bacteria that penetrate into this normally aseptic site. Examination of the mucosa in ileal CD has been informative. It is here that the density of $E$. coli, enriched in virulence and secretion pathways, is elevated as determined by culture and molecular methods $(11,22$, 57). Use of bowel-specific aminosalicylates is associated with normalization of this E. coli bloom in ileal disease $(22,58)$, suggesting that inflammation might somehow benefit certain bacteria that are associated with the mucosal surface. This finding is important clinically because the severity of ileal disease has been shown to correlate directly with the density of mucosa-associated E. coli (12). The implication of course is that reducing the burden of mucosaassociated bacteria would have favorable outcomes on the disease course, possibly opening up new therapeutic avenues beyond immunosuppression. Together, these data support the general view that mucosa-associated $E$. coli are of pathogenic significance in the disease and that their abundance can be manipulated using pharmacologic treatments directed at host inflammation. These results stimulate many important and interesting questions. For example, they imply that the selective environment favoring $E$. coli expansion at the mucosal surface is inflammatory in nature. Although the exact source of this selection is not yet known, fruitful lines of investigation can be envisaged to uncover it. Furthermore, does the inflamed mucosa render a host more susceptible to de novo colonization by bacteria with pathobiont-like features, or is this expansion seeded from a resident population of opportunistic bacteria that exploit this favorable niche? Lastly, the genetically encoded bacterial adaptations needed for mucosal expansion in the inflamed gut are not known, but uncovering them would offer valuable insight into the evolutionary process that selects for such pathobionts. The upshot of this information would be new useful genetic markers for detecting pathobionts in hosts and other potential environmental reservoirs, and in identifying new potential targets for antimicrobial drug discovery.

\section{ADHERENT-INVASIVE E. coli IN CROHN'S DISEASE}

Much experimental and observational data implicate infectious agents in the initiation and maintenance of chronic inflammation in the intestine (6). E. coli is a Gram-negative species in the intestine where it can have a positive effect on gut homeostasis. However, through acquisition of virulence factors, such as toxins, adhesins, and secretion systems, E. coli can develop pathogenic traits that participate in intestinal and extraintestinal disease processes (59). Work originating from the laboratory of Dr. Darfeuille-Michaud identified E. coli in ileal biopsies from patients with CD (57), which they called adherent-invasive E. coli (AIEC) to reflect their atypical ability to adhere to mucosal epithelial cells and invade and survive within human cells. These E. coli were found to lack known virulence factors typical of pathogenic $E$. coli, including type III secretion systems or phage-encoded toxins, suggesting that they were a newly described pathovar. A series of papers followed that detailed the intracellular lifestyle of AIEC and the inflammatory response by cultured cells to AIEC infection (60, 61). AIEC are capable of replicating within macrophages and causing these cells to release high amounts of TNF $\alpha$ in vitro (62). It also appears possible that AIEC exploit cell phenotypes associated with genetic CD risk factors, such as defects in autophagy (63).

Several studies have now confirmed that AIEC are enriched in humans with CD, where they are six-times more likely to be isolated from ileal and colonic samples compared to healthy controls and represent the dominant bacterial species present $(57,64,65)$. A growing body of work has uncovered genetic and phenotypic diversity among AIEC isolated from adults (12, 65-67), children $(68,69)$, and companion animals (70) indicating that different host environments can select for the AIEC phenotype. Since AIEC can be isolated from seemingly healthy individuals (albeit much less frequently than in Crohn's), this genetic diversity suggests that interactions between AIEC and other CD risk factors might be needed to elicit disease in a subset of individuals.

In 2013, Small et al. established a novel model for chronic AIEC infection in conventional mice (8), that over time, develop transmural inflammation and fibrosis in the small and large intestine, the very hallmarks of CD. Importantly, this happens over timescales consistent with a progressive chronic disease without the need for foreign chemicals to induce inflammation. Using this model, it was shown that AIEC is resistant to innate antimicrobial defenses at the epithelial surface, including resistance to antimicrobial peptides released by Paneth cells and colonocytes (71). The molecular mechanism for this resistance involves, in part, the expression of a surface-localized protease that cleaves cationic host defense peptides to render them inactive. In addition to resistance to host-derived antimicrobial molecules, AIEC tend to be resistant to multiple xenobiotic antimicrobials, particularly in isolates obtained from patients with ileal disease (72). This likely reflects the selective environment in which AIEC evolve within hosts, as $\mathrm{CD}$ patients are often given antibiotics as part of their treatment regimen. As of yet, no studies have examined whether eradicating AIEC is a viable or efficacious strategy in the treatment of CD; however, animal models permitting chronic colonization and disease progression will help facilitate such studies in the future.

\section{CONCLUSION}

Intestinal bacteria play an important role in the pathogenesis of CD in a manner that is incompletely understood. External risk factors, such as acute infectious gastroenteritis and antibiotic exposure, are 
moderately associated with $\mathrm{CD}$, yet neither can be considered sufficient to elicit disease on their own. It is likely that a combination of genetic and environmental risk factors is necessary in the pathogenesis of CD; however, exactly how such a constellation of risk factors manifest in disease expression has been a challenging problem to address. The study of mucosal-associated E. coli with pathobiont-like properties has yielded promising new leads into the pathogenesis of $\mathrm{CD}$ but more work needs to be done to understand where such microbes come from, where they evolve their pathogenic features, and what the effect of their eradication might have on susceptible hosts. A mechanistic understanding of the pathogenic microbes in CD will offer more therapeutic targets and perhaps paradigm shifting approaches toward curative treatments for this disease.

\section{REFERENCES}

1. Bernstein CN, Wajda A, Svenson LW, Mackenzie A, Koehoorn M, Jackson $\mathrm{M}$, et al. The epidemiology of inflammatory bowel disease in Canada: a population-based study. Am J Gastroenterol (2006) 101:1559-68. doi:10.1111/j.1572-0241.2006.00603.x

2. Kappelman MD, Rifas-Shiman SL, Porter CQ, Ollendorf DA, Sandler RS, Galanko JA, et al. Direct health care costs of Crohn's disease and ulcerative colitis in US children and adults. Gastroenterology (2008) 135:1907-13. doi:10.1053/j.gastro.2008.09.012

3. Van Limbergen J, Wilson DC, Satsangi J. The genetics of Crohn's disease. Annu Rev Genomics Hum Genet (2009) 10:89-116. doi:10.1146/ annurev-genom-082908-150013

4. Franke A, Mcgovern DP, Barrett JC, Wang K, Radford-Smith GL, Ahmad T, et al. Genome-wide meta-analysis increases to 71 the number of confirmed Crohn's disease susceptibility loci. Nat Genet (2010) 42:1118-25. doi:10.1038/ ng.717

5. Baumgart DC, Sandborn WJ. Crohn's disease. Lancet (2012) 380:1590-605. doi:10.1016/S0140-6736(12)60026-9

6. Sartor RB. Microbial influences in inflammatory bowel diseases. Gastroenterology (2008) 134:577-94. doi:10.1053/j.gastro.2007.11.059

7. Carvalho FA, Barnich N, Sivignon A, Darcha C, Chan CH, Stanners CP, et al. Crohn's disease adherent-invasive Escherichia coli colonize and induce strong gut inflammation in transgenic mice expressing human CEACAM. J Exp Med (2009) 206:2179-89. doi:10.1084/jem.20090741

8. Small CL, Reid-Yu SA, Mcphee JB, Coombes BK. Persistent infection with Crohn's disease-associated adherent-invasive Escherichia coli leads to chronic inflammation and intestinal fibrosis. Nat Commun (2013) 4:1957. doi:10.1038/ ncomms 2957

9. Wlodarska M, Kostic AD, Xavier RJ. An integrative view of microbiome-host interactions in inflammatory bowel diseases. Cell Host Microbe (2015) 17:577-91. doi:10.1016/j.chom.2015.04.008

10. D’Haens GR, Geboes K, Peeters M, Baert F, Penninckx F, Rutgeerts P. Early lesions of recurrent Crohn's disease caused by infusion of intestinal contents in excluded ileum. Gastroenterology (1998) 114:262-7. doi:10.1016/ S0016-5085(98)70476-7

11. Swidsinski A, Ladhoff A, Pernthaler A, Swidsinski S, Loening-Baucke V, Ortner $\mathrm{M}$, et al. Mucosal flora in inflammatory bowel disease. Gastroenterology (2002) 122:44-54. doi:10.1053/gast.2002.30294

12. Baumgart M, Dogan B, Rishniw M, Weitzman G, Bosworth B, Yantiss R, et al. Culture independent analysis of ileal mucosa reveals a selective increase in invasive Escherichia coli of novel phylogeny relative to depletion of Clostridiales in Crohn's disease involving the ileum. ISME J (2007) 1:403-18. doi:10.1038/ismej.2007.52

13. Manichanh C, Borruel N, Casellas F, Guarner F. The gut microbiota in IBD. Nat Rev Gastroenterol Hepatol (2012) 9:599-608. doi:10.1038/nrgastro.2012.152

14. Vaahtovuo J, Korkeamaki M, Munukka E, Viljanen MK, Toivanen P. Quantification of bacteria in human feces using 16S rRNA-hybridization, DNA-staining and flow cytometry. J Microbiol Methods (2005) 63:276-86. doi:10.1016/j.mimet.2005.03.017

\section{AUTHOR CONTRIBUTIONS}

$\mathrm{AO}$ and $\mathrm{BC}$ wrote the paper.

\section{ACKNOWLEDGMENTS}

We are grateful to members of the Coombes lab for helpful discussions. This work was supported by grants from the Canadian Institutes of Health Research (MOP-136968) and Crohn's and Colitis Canada. AO is supported by a Canada Graduate Scholarship from the Canadian Institutes of Health Research. BC is the Canada Research Chair in Infectious Disease Pathogenesis.

15. Kuczynski J, Stombaugh J, Walters WA, Gonzalez A, Caporaso JG, Knight R. Using QIIME to analyze 16S rRNA gene sequences from microbial communities. Curr Protoc Bioinformatics (2011) 10:17. doi:10.1002/0471250953. bi1007s36

16. Liu Z, Desantis TZ, Andersen GL, Knight R. Accurate taxonomy assignments from 16S rRNA sequences produced by highly parallel pyrosequencers. Nucleic Acids Res (2008) 36:e120. doi:10.1093/nar/gkn491

17. Manichanh C, Rigottier-Gois L, Bonnaud E, Gloux K, Pelletier E, Frangeul L, et al. Reduced diversity of faecal microbiota in Crohn's disease revealed by a metagenomic approach. Gut (2006) 55:205-11. doi:10.1136/gut.2005.073817

18. Topping DL, Clifton PM. Short-chain fatty acids and human colonic function: roles of resistant starch and nonstarch polysaccharides. Physiol Rev (2001) 81:1031-64.

19. Vogt SL, Pena-Diaz J, Finlay BB. Chemical communication in the gut: effects of microbiota-generated metabolites on gastrointestinal bacterial pathogens. Anaerobe (2015) 34:106-15. doi:10.1016/j.anaerobe.2015.05.002

20. Sokol H, Seksik P, Rigottier-Gois L, Lay C, Lepage P, Podglajen I, et al. Specificities of the fecal microbiota in inflammatory bowel disease. Inflamm Bowel Dis (2006) 12:106-11. doi:10.1097/01.MIB.0000200323.38139.c6

21. Frank DN, St Amand AL, Feldman RA, Boedeker EC, Harpaz N, Pace NR. Molecular-phylogenetic characterization of microbial community imbalances in human inflammatory bowel diseases. Proc Natl Acad Sci U S A (2007) 104:13780-5. doi:10.1073/pnas.0706625104

22. Morgan XC, Tickle TL, Sokol H, Gevers D, Devaney KL, Ward DV, et al. Dysfunction of the intestinal microbiome in inflammatory bowel disease and treatment. Genome Biol (2012) 13:R79. doi:10.1186/gb-2012-13-9-r79

23. Gaboriau-Routhiau V, Rakotobe S, Lecuyer E, Mulder I, Lan A, Bridonneau $\mathrm{C}$, et al. The key role of segmented filamentous bacteria in the coordinated maturation of gut helper $\mathrm{T}$ cell responses. Immunity (2009) 31:677-89. doi:10.1016/j.immuni.2009.08.020

24. Sczesnak A, Segata N, Qin X, Gevers D, Petrosino JF, Huttenhower C, et al. The genome of th17 cell-inducing segmented filamentous bacteria reveals extensive auxotrophy and adaptations to the intestinal environment. Cell Host Microbe (2011) 10:260-72. doi:10.1016/j.chom.2011.08.005

25. Keshavarzian A, Banan A, Farhadi A, Komanduri S, Mutlu E, Zhang Y, et al. Increases in free radicals and cytoskeletal protein oxidation and nitration in the colon of patients with inflammatory bowel disease. Gut (2003) 52:720-8. doi:10.1136/gut.52.5.720

26. Sandkvist M. Type II secretion and pathogenesis. Infect Immun (2001) 69:3523-35. doi:10.1128/IAI.69.6.3523-3535.2001

27. Gevers D, Kugathasan S, Denson LA, Vazquez-Baeza Y, Van Treuren W, Ren B, et al. The treatment-naive microbiome in new-onset Crohn's disease. Cell Host Microbe (2014) 15:382-92. doi:10.1016/j.chom.2014.02.005

28. Lahad A, Weiss B. Current therapy of pediatric Crohn's disease. World J Gastrointest Pathophysiol (2015) 6:33-42. doi:10.4291/wjgp.v6.i2.33

29. Su JW, Ma JJ, Zhang HJ. Use of antibiotics in patients with Crohn's disease: a systematic review and meta-analysis. J Dig Dis (2015) 16:58-66. doi:10.1111/1751-2980.12216

30. Schaubeck M, Clavel T, Calasan J, Lagkouvardos I, Haange SB, Jehmlich N, et al. Dysbiotic gut microbiota causes transmissible Crohn's disease-like ileitis 
independent of failure in antimicrobial defence. Gut (2015). doi:10.1136/ gutjnl-2015-309333

31. Hansen R, Russell RK, Reiff C, Louis P, Mcintosh F, Berry SH, et al. Microbiota of de-novo pediatric IBD: increased Faecalibacterium prausnitzii and reduced bacterial diversity in Crohn's but not in ulcerative colitis. Am J Gastroenterol (2012) 107:1913-22. doi:10.1038/ajg.2012.335

32. Sokol H, Seksik P, Furet JP, Firmesse O, Nion-Larmurier I, Beaugerie L, et al. Low counts of Faecalibacterium prausnitzii in colitis microbiota. Inflamm Bowel Dis (2009) 15:1183-9. doi:10.1002/ibd.20903

33. Sokol H, Pigneur B, Watterlot L, Lakhdari O, Bermudez-Humaran LG, Gratadoux JJ, et al. Faecalibacterium prausnitzii is an anti-inflammatory commensal bacterium identified by gut microbiota analysis of Crohn disease patients. Proc Natl Acad Sci U S A (2008) 105:16731-6. doi:10.1073/ pnas. 0804812105

34. Martin R, Chain F, Miquel S, Lu J, Gratadoux JJ, Sokol H, et al. The commensal bacterium Faecalibacterium prausnitzii is protective in DNBS-induced chronic moderate and severe colitis models. Inflamm Bowel Dis (2014) 20:417-30. doi:10.1097/01.MIB.0000440815.76627.64

35. Quevrain E, Maubert MA, Michon C, Chain F, Marquant R, Tailhades J, et al. Identification of an anti-inflammatory protein from Faecalibacterium prausnitzii, a commensal bacterium deficient in Crohn's disease. Gut (2015). doi:10.1136/gutjnl-2014-307649

36. Garcia Rodriguez LA, Ruigomez A, Panes J. Acute gastroenteritis is followed by an increased risk of inflammatory bowel disease. Gastroenterology (2006) 130:1588-94. doi:10.1053/j.gastro.2006.02.004

37. Porter CK, Tribble DR, Aliaga PA, Halvorson HA, Riddle MS. Infectious gastroenteritis and risk of developing inflammatory bowel disease. Gastroenterology (2008) 135:781-6. doi:10.1053/j.gastro.2008.05.081

38. Gradel KO, Nielsen HL, Schonheyder HC, Ejlertsen T, Kristensen B, Nielsen $H$. Increased short- and long-term risk of inflammatory bowel disease after Salmonella or Campylobacter gastroenteritis. Gastroenterology (2009) 137:495-501. doi:10.1053/j.gastro.2009.04.001

39. Stecher B, Robbiani R, Walker AW, Westendorf AM, Barthel M, Kremer M, et al. Salmonella enterica serovar typhimurium exploits inflammation to compete with the intestinal microbiota. PLoS Biol (2007) 5:2177-89. doi:10.1371/ journal.pbio.0050244

40. Pop M, Walker AW, Paulson J, Lindsay B, Antonio M, Hossain MA, et al. Diarrhea in young children from low-income countries leads to large-scale alterations in intestinal microbiota composition. Genome Biol (2014) 15:R76. doi:10.1186/gb-2014-15-6-r76

41. David LA, Weil A, Ryan ET, Calderwood SB, Harris JB, Chowdhury F, et al. Gut microbial succession follows acute secretory diarrhea in humans. MBio (2015) 6:e381-315. doi:10.1128/mBio.00381-15

42. Mai V, Braden CR, Heckendorf J, Pironis B, Hirshon JM. Monitoring of stool microbiota in subjects with diarrhea indicates distortions in composition. $J$ Clin Microbiol (2006) 44:4550-2. doi:10.1128/JCM.01542-06

43. Lupp C, Robertson ML, Wickham ME, Sekirov I, Champion OL, Gaynor EC, et al. Host-mediated inflammation disrupts the intestinal microbiota and promotes the overgrowth of Enterobacteriaceae. Cell Host Microbe (2007) 2:119-29. doi:10.1016/j.chom.2007.06.010

44. Winter SE, Thiennimitr P, Winter MG, Butler BP, Huseby DL, Crawford RW, et al. Gut inflammation provides a respiratory electron acceptor for Salmonella. Nature (2010) 467:426-9. doi:10.1038/nature09415

45. Khan KJ, Ullman TA, Ford AC, Abreu MT, Abadir A, Marshall JK, et al. Antibiotic therapy in inflammatory bowel disease: a systematic review and meta-analysis. Am J Gastroenterol (2011) 106:661-73. doi:10.1038/ajg.2011.72

46. Pepin J, Saheb N, Coulombe MA, Alary ME, Corriveau MP, Authier S, et al. Emergence of fluoroquinolones as the predominant risk factor for Clostridium difficile-associated diarrhea: a cohort study during an epidemic in Quebec. Clin Infect Dis (2005) 41:1254-60. doi:10.1086/496986

47. Dethlefsen L, Huse S, Sogin ML, Relman DA. The pervasive effects of an antibiotic on the human gut microbiota, as revealed by deep 16S rRNA sequencing. PLoS Biol (2008) 6:e280. doi:10.1371/journal.pbio.0060280

48. Shaw SY, Blanchard JF, Bernstein CN. Association between the use of antibiotics and new diagnoses of Crohn's disease and ulcerative colitis. Am J Gastroenterol (2011) 106:2133-42. doi:10.1038/ajg.2011.304
49. Card T, Logan RF, Rodrigues LC, Wheeler JG. Antibiotic use and the development of Crohn's disease. Gut (2004) 53:246-50. doi:10.1136/gut.2003.025239

50. Margolis DJ, Fanelli M, Hoffstad O, Lewis JD. Potential association between the oral tetracycline class of antimicrobials used to treat acne and inflammatory bowel disease. Am J Gastroenterol (2010) 105:2610-6. doi:10.1038/ ajg. 2010.303

51. Hviid A, Svanstrom H, Frisch M. Antibiotic use and inflammatory bowel diseases in childhood. Gut (2011) 60:49-54. doi:10.1136/gut.2010.219683

52. Virta L, Auvinen A, Helenius H, Huovinen P, Kolho KL. Association of repeated exposure to antibiotics with the development of pediatric Crohn's disease - a nationwide, register-based Finnish case-control study. Am J Epidemiol (2012) 175:775-84. doi:10.1093/aje/kwr400

53. Hildebrand H, Malmborg P, Askling J, Ekbom A, Montgomery SM. Early-life exposures associated with antibiotic use and risk of subsequent Crohn's disease. Scand J Gastroenterol (2008) 43:961-6. doi:10.1080/00365520801971736

54. Aberra FN, Brensinger CM, Bilker WB, Lichtenstein GR, Lewis JD. Antibiotic use and the risk of flare of inflammatory bowel disease. Clin Gastroenterol Hepatol (2005) 3:459-65. doi:10.1016/S1542-3565(05)00020-0

55. Jakobsson HE, Jernberg C, Andersson AF, Sjolund-Karlsson M, Jansson JK, Engstrand L. Short-term antibiotic treatment has differing long-term impacts on the human throat and gut microbiome. PLoS One (2010) 5:e9836. doi:10.1371/journal.pone.0009836

56. Kaser A, Zeissig S, Blumberg RS. Inflammatory bowel disease. Annu Rev Immunol (2010) 28:573-621. doi:10.1146/annurev-immunol-030409-101225

57. Darfeuille-Michaud A, Neut C, Barnich N, Lederman E, Di Martino P, Desreumaux P, et al. Presence of adherent Escherichia coli strains in ileal mucosa of patients with Crohn's disease. Gastroenterology (1998) 115:1405-13. doi:10.1016/S0016-5085(98)70019-8

58. Benjamin JL, Hedin CR, Koutsoumpas A, Ng SC, Mccarthy NE, Prescott NJ, et al. Smokers with active Crohn's disease have a clinically relevant dysbiosis of the gastrointestinal microbiota. Inflamm Bowel Dis (2012) 18:1092-100. doi:10.1002/ibd.21864

59. Croxen MA, Finlay BB. Molecular mechanisms of Escherichia coli pathogenicity. Nat Rev Microbiol (2010) 8:26-38. doi:10.1038/nrmicro2265

60. Barnich N, Darfeuille-Michaud A. Adherent-invasive Escherichia coli and Crohn's disease. Curr Opin Gastroenterol (2007) 23:16-20. doi:10.1097/ MOG.0b013e3280105a38

61. Chassaing B, Darfeuille-Michaud A. The commensal microbiota and enteropathogens in the pathogenesis of inflammatory bowel diseases. Gastroenterology (2011) 140:1720-8. doi:10.1053/j.gastro.2011.01.054

62. Glasser AL, Boudeau J, Barnich N, Perruchot MH, Colombel JF, DarfeuilleMichaud A. Adherent invasive Escherichia coli strains from patients with Crohn's disease survive and replicate within macrophages without inducing host cell death. Infect Immun (2001) 69:5529-37. doi:10.1128/ IAI.69.9.5529-5537.2001

63. Lapaquette P, Glasser AL, Huett A, Xavier RJ, Darfeuille-Michaud A. Crohn's disease-associated adherent-invasive E. coli are selectively favoured by impaired autophagy to replicate intracellularly. Cell Microbiol (2010) 12:99-113. doi:10.1111/j.1462-5822.2009.01381.x

64. Darfeuille-Michaud A, Boudeau J, Bulois P, Neut C, Glasser AL, Barnich $\mathrm{N}$, et al. High prevalence of adherent-invasive Escherichia coli associated with ileal mucosa in Crohn's disease. Gastroenterology (2004) 127:412-21. doi:10.1053/j.gastro.2004.04.061

65. Martinez-Medina M, Aldeguer X, Lopez-Siles M, Gonzalez-Huix F, LopezOliu C, Dahbi G, et al. Molecular diversity of Escherichia coli in the human gut: new ecological evidence supporting the role of adherent-invasive $E$. coli (AIEC) in Crohn's disease. Inflamm Bowel Dis (2009) 15:872-82. doi:10.1002/ ibd. 20860

66. Nash JH, Villegas A, Kropinski AM, Aguilar-Valenzuela R, Konczy P, Mascarenhas M, et al. Genome sequence of adherent-invasive Escherichia coli and comparative genomic analysis with other E. coli pathotypes. BMC Genomics (2010) 11:667. doi:10.1186/1471-2164-11-667

67. Sepehri S, Khafipour E, Bernstein CN, Coombes BK, Pilar AV, Karmali M, et al. Characterization of Escherichia coli isolated from gut biopsies of newly diagnosed patients with inflammatory bowel disease. Inflamm Bowel Dis (2011) 17:1451-63. doi:10.1002/ibd.21509 
68. Negroni A, Costanzo M, Vitali R, Superti F, Bertuccini L, Tinari A, et al. Characterization of adherent-invasive Escherichia coli isolated from pediatric patients with inflammatory bowel disease. Inflamm Bowel Dis (2012) 18:913-24. doi:10.1002/ibd.21899

69. Schippa S, Iebba V, Totino V, Santangelo F, Lepanto M, Alessandri C, et al. A potential role of Escherichia coli pathobionts in the pathogenesis of pediatric inflammatory bowel disease. Can J Microbiol (2012) 58:426-32. doi:10.1139/ w2012-007

70. Simpson KW, Dogan B, Rishniw M, Goldstein RE, Klaessig S, Mcdonough PL, et al. Adherent and invasive Escherichia coli is associated with granulomatous colitis in boxer dogs. Infect Immun (2006) 74:4778-92. doi:10.1128/ IAI.00067-06

71. McPhee JB, Small CL, Reid-Yu SA, Brannon JR, Le Moual H, Coombes BK. Host defense peptide resistance contributes to colonization and maximal intestinal pathology by Crohn's disease-associated adherent-invasive Escherichia coli. Infect Immun (2014) 82:3383-93. doi:10.1128/IAI.01888-14
72. Dogan B, Scherl E, Bosworth B, Yantiss R, Altier C, Mcdonough PL, et al. Multidrug resistance is common in Escherichia coli associated with ileal Crohn's disease. Inflamm Bowel Dis (2013) 19:141-50. doi:10.1002/ ibd.22971

Conflict of Interest Statement: The authors declare that the research was conducted in the absence of any commercial or financial relationships that could be construed as a potential conflict of interest.

Copyright $\odot 2015$ Oberc and Coombes. This is an open-access article distributed under the terms of the Creative Commons Attribution License (CC BY). The use, distribution or reproduction in other forums is permitted, provided the original author(s) or licensor are credited and that the original publication in this journal is cited, in accordance with accepted academic practice. No use, distribution or reproduction is permitted which does not comply with these terms. 